\title{
In-situ analysis of strain localization related to structural heterogeneities of carbonate rocks
}

\author{
J. Dautriat ${ }^{1,2}$, M. Bornert ${ }^{1,3, a}$, N. Gland ${ }^{2}$, A. Dimanov ${ }^{1}$, and J. Raphanel ${ }^{1}$ \\ ${ }^{1}$ Laboratoire de Mécanique des Solides, 91128, Palaiseau, France. \\ ${ }^{2}$ Institut Français du Pétrole IFP, 92501, Rueil-Malmaison, France. \\ ${ }^{3}$ Unité Navier, École des Ponts ParisTech, 77455 Marne-la-Vallée Cedex, France
}

The technique of Digital Image Correlation (DIC) has been applied to study the deformation of porous carbonate rocks subjected to uniaxial compression tests. The tests have been performed at two different scales: on cylinders of $10 \mathrm{~cm}$ high compressed with a standard press with digital images recorded by optical microscopy at a global and local scale and on smaller parallelepiped samples deformed inside a scanning electron microscope (SEM). The development of localization at different scales is thus recorded as well as the damage and compaction mechanisms in relation with the microstructural heterogeneities.

\section{Introduction}

Digital Image Correlation (DIC) is a powerful method which allows the determination of the complete deformation field of materials during mechanical testing. It is particularly appropriate for heterogeneous materials which are prone to localization or damage ([1], [2]). This technique however has not been widely used for geomaterials, which present microstructural heterogeneities at different scales, but have the drawback of sustaining relatively small deformation before fracture ([3]-[6]). In this study, we deform samples of Estaillades carbonate by uniaxial compression. This carbonate is composed of $99 \%$ calcite with grains between 1 and 10 micrometers which form two types of aggregates : dense and microporous with characteristics sizes between 100 and $500 \mu \mathrm{m}$. This leads to a bimodal porosity, an inter-aggregate mesoporosity and an intra-aggregate microporosity with respective pore thresholds of 1 and 0.3 micrometers. The porosity of the tested samples varies between $24 \%$ and $31 \%$ owing to the heterogeneous distributions of both kinds of aggregates. Hydromechanical measures and post-mortem observations indicate indirectly that these microstructural heterogeneities play a role for the selective activation of damage mechanisms, variations of directional permeabilities and compressibility ([7], [8]). DIC allows the computation of strain fields at different stages of the deformation history and thus provides some insight on the history of localization or damage development. It will be applied here to macroscopic samples and also to smaller samples, deformed by compression inside the chamber of an environmental scanning electron microscope ([9]).

To our knowledge, it is the first time that DIC is applied to this class of geomaterials which maximum global axial strain before fracture does not exceed $0.5 \%$. We have performed two types of uniaxial compression tests. At fist, cylindrical samples (height $10 \mathrm{~cm}$,

\footnotetext{
${ }^{\mathrm{a}}$ Email : michel.bornert@enpc.fr
} 
diameter $5 \mathrm{~cm}$ ) are prepared with two opposite flat surfaces machined along its height for the purpose of image recording, and placed within a standard electromechanical press, which is operated in displacement control. Two optical cameras (one on each side) record digital images over the length of the whole sample and over a selected smaller zone $\left(7.5 \times 7.5 \mathrm{~mm}^{2}\right)$, respectively, thus providing data at the macroscopic and mesoscopic scales. Secondly, centimeter-sized samples $\left(10 \times 5 \times 5 \mathrm{~mm}^{3}\right)$ are uniaxially deformed inside a scanning electron microscope (SEM), and digital images are recorded during loading. The size of the observed area is $3 \times 2 \mathrm{~mm}^{2}$ and represents our microscopic scale.

\section{DIC technique and application to different scales of observation}

\subsection{Main features of DIC applied to measure deformation fields}

This method allows the determination of the deformation and strain fields at different scales by comparing a reference image and a current image at a deformed stage, without contact or modification of the observed surface. The main condition is that the observed surface presents enough contrasts to generate appropriate grey level fluctuations. When the natural contrast is not enough, it may be enhanced by spray paint, taking care that the droplets are small. The DIC procedure consists in finding the homologous positions of a set of points in two or more images by minimizing a correlation coefficient which measures the similarity of the grey level distribution in a small domain around these points, assuming that this distribution is converted by the material transformation according to optical flow conservation principles ([3]). A domain $\mathrm{D}$ is thus transformed into $\Phi(\mathrm{D})$, the local transformation is taken as a rigid body motion (translation plus rotation) coupled with an homogeneous deformation whose gradient is assumed to be equal to the macroscopic transformation gradient. The minimization of the correlation coefficient is performed in an automated way by the software "CorrelManuV" ([10]), which then allows an estimation of the local displacements with a sub-pixel resolution, the strain field is then computed by considering neighboring points around the selected point, at small strains a compromise has to be made between locality and precision ([10]- [12]).

The magnitude of components of the deformation field is represented on maps superimposed over the picture of the microstructure. From the deformation field, one may compute the components of the strain tensor and also the equivalent Von Mises strain. The principal strains are represented as a cross whose branches are aligned with the major principal direction of strain and a color code indicates the magnitude of the equivalent strain.

\subsection{Optical tests}

The uniaxial deformation tests are run in displacement control mode with a classical electromechanical press. An LVDT and a load cell attached to the loading piston allow Monitoring of displacement and load, respectively. A displacement rate of $1 \mu \mathrm{m} \mathrm{s}^{-1}$ (axial strain rate of the order of $10^{-6} \mathrm{~s}^{-1}$ ) is applied until sample fracture, which occurs for an average axial compression stress of $15 \mathrm{MPa}$. During a test which lasts about 10 minutes, an average of 250 pictures are taken at two different scales by two cameras aiming at two sides of the sample. A CCD Imperx Camera (16 MPixels) records pictures over the whole height of the sample (pixel size 22 micrometers) and a CCD Spot Camera (4MPixel) takes pictures of a $7.5 \times 7.5 \mathrm{~mm}^{2}$ zone (pixel size 3.5 micrometer). The natural contrast of the samples has been enhanced by the use of spray paint. The droplets should be smaller than a pixel, but we have not been able to do better than 10 to 100 micrometers which covers a few pixels. Great care is taken so that the cameras axes are perfectly orthogonal to the flat surfaces of the samples. The lightning of the surfaces is also optimized to provide the wider histogram of grey levels. 


\subsection{SEM tests}

The chamber of the FEI Environmental Scanning Microscope Qanta600 has been specially equipped with a miniaturized simple compression device. The tests are performed at a minimum deformation rate of 1 micrometer per second. The image recording times are fairly long (several minutes), which requires the mechanical testing to be made in a step by step mode and limits the number of pictures for a given test to about 5 . The natural contrast is enough for image correlation using secondary electron imaging. Contrast and brightness are adjusted before each test so that the full range of 256 grey levels is covered without saturation. Beam size and scanning rate are adjusted for the best compromise between the quality of the signal to noise ratio and the problem of electrical charge building. Magnifications are between 40 and 160 . With a magnification of 40 , the picture covers about one quarter of the sample $\left(3.7 \times 3.4 \mathrm{~mm}^{2}\right)$ for a resolution of $910 \mathrm{~nm}$ per pixel. This scale allows the observation of local phenomena which may involve the whole sample. The higher magnification ( $230 \mathrm{~nm}$ per pixel) provides access to a smaller local scale. The observed side must be carefully polished (up to SiC paper, grade 4000) and coated with gold.

\section{Results}

The results may be sorted according to the scale of observation, the macroscopic and mesoscopic ones recorded by optical microscopy and the microscopic one recorded in the SEM.

\subsection{Macroscopic and mesoscopic observations}

Figure 1 shows results from macroscopic observations. Figure 1.a shows the stress-strain curves: axial strain computed from the LVDT signal and axial and transverse strain computed from DIC over the whole length (green and red) or over a smaller area (blue). Young's modulus from the LVDT curve is estimated at $6 \mathrm{GPa}$, while the DIC computation yields 13 GPa and a Poisson's ratio of 0.25 . The LVDT also overestimates the axial strain since the measure includes the initial sample setting and the rigidity of the testing device. The macroscopic images show an initial homogeneous strain up to about $\varepsilon_{11}=0.12 \%$ followed by the development of a lens shaped region of deformation for $\varepsilon_{11}=0.20 \%$ (Fig1. Image A) which grows and generates a strain gradient along the sample (Fig1. Image B). At this stage, the mean axial strain is about $0.30 \%$, but the equivalent strain is about ten times larger at the top than at the bottom of the sample. Near the peak stress, at a mean axial strain of $0.37 \%$, a zone of very intense strain localization is shown by DIC, which anticipates the position of macroscopic fracture (Fig1. Image C). An analysis of microstructures along the sample shows that clusters of dense aggregates are found in the central part of the sample which is the site of fracture propagation, whereas in the lower part, the dense and micro-porous aggregates are more homogeneously distributed.

Figure 2 shows results of mesoscopic observation. The observed area has been chosen for its heterogeneous character, with its lower and upper part consisting mostly of dense aggregates and the central part being exclusively filled by micro-porous aggregates. The Figure 2 shows the macroscopic monitoring of stress and strain and the computed stressstrain curves, and the corresponding images (from A to D), showing the progressive evolution of strain obtained by DIC for the observed area. After homogeneous initial deformation, there is no localization, but rather diffuse and progressive opening of microcracks in-between the dense aggregates (image A), finally oriented at nearly fixed angle 
in respect to the loading direction. These cracks then propagate through the micro-porous aggregates (B), intensify near the peak stress (C) and coalesce (D) leading to relaxation and macroscopic failure.
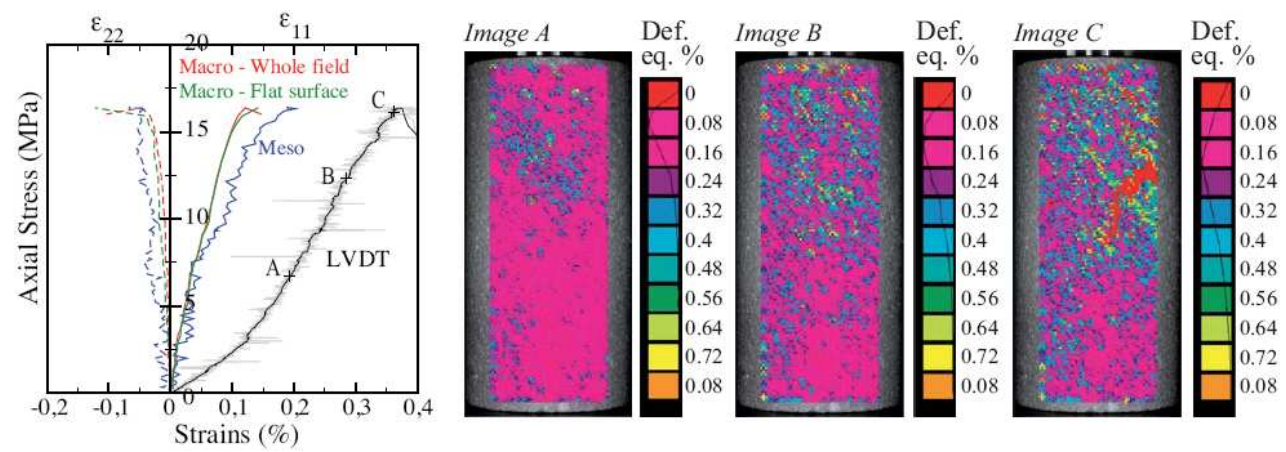

Fig. 1. Stress-strain curves present the LVDT measures (note the lower estimate of E) and the computed axial and radial strains from DIC at different scales (whole sample scale in red, whole flat surface area in green, a limited central area in blue). The maps of equivalent strains (corresponding to the whole flat surface area) are superimposed over the sample picture for 3 strain levels measured by LVDT as $0.2,0.29$ and $0.37 \%$ respectively. Image C is just before macroscopic fracture.
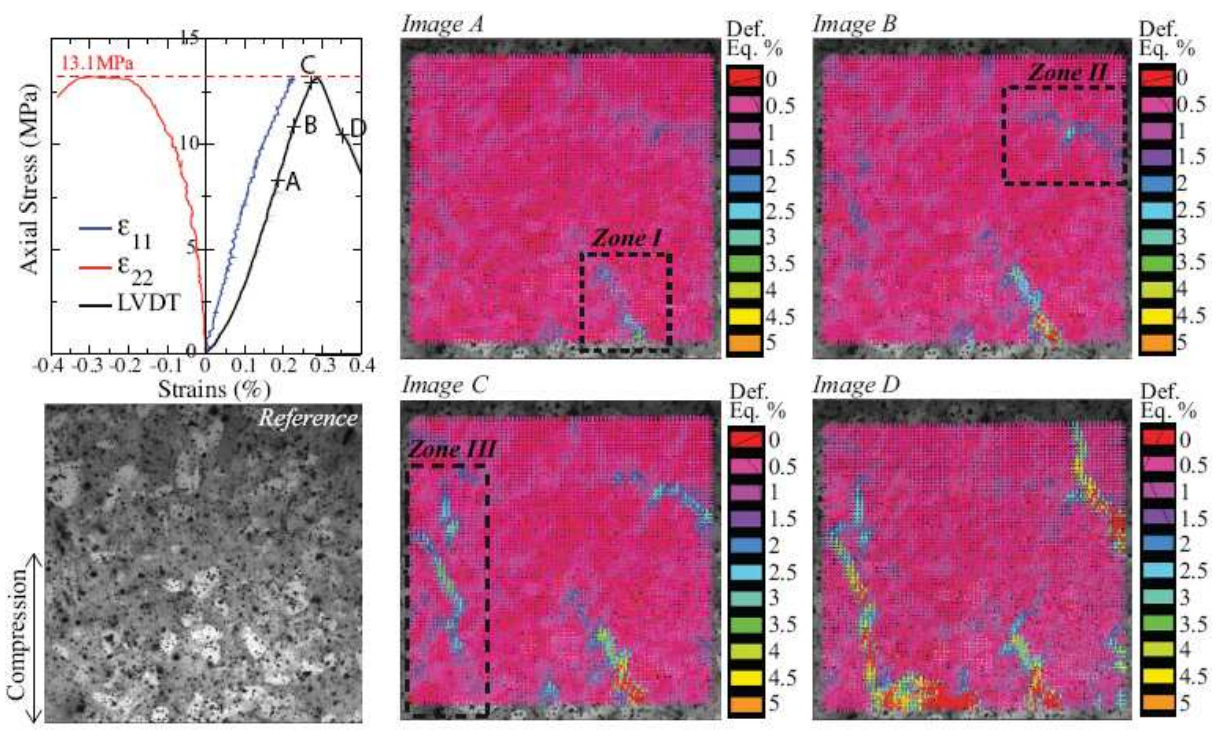

Fig. 2. Stress-strain curves (LVDT measure in black and computed measures in colour), reference micrograph of the observed area (paint spray in appears in black, dense and porous aggregates appear in white and grey, respectively) and maps of equivalent strains at the 4 steps marked on the stressstrain curve (Measure basis of 36 pixels).

\subsection{Microscopic tests}

If the size of the observed region is of same the order as the one observed at the mesoscopic scale, the image resolution is much finer, so that more details of the microstructures can be resolved. Owing to the time necessary to store an image, the test is carried out in a step by step procedure. At each step a very slight relaxation was observed. 
Figure 3 shows SEM pictures from the selected area at two scales, and below each one the corresponding equivalent deformation maps at 3 successive load steps. The localization appears at the first step at the interfaces of dense aggregates (dark grey on the micrograph) which are zones of larger porosity. It evolves mostly as a microcrack opening which propagates in a wavy pattern, following the geometry of the dense aggregates (left-hand images). At finer scale, localizations appears at interfaces in-between dense aggregates, but very little localized events take place through the microporous aggregates of the zone of observation (right-hand images). The propagation seems to occur in a gradual manner, along the regions of lowest strength; the local relative motions are larger in the most torturous zone of the microcrack.

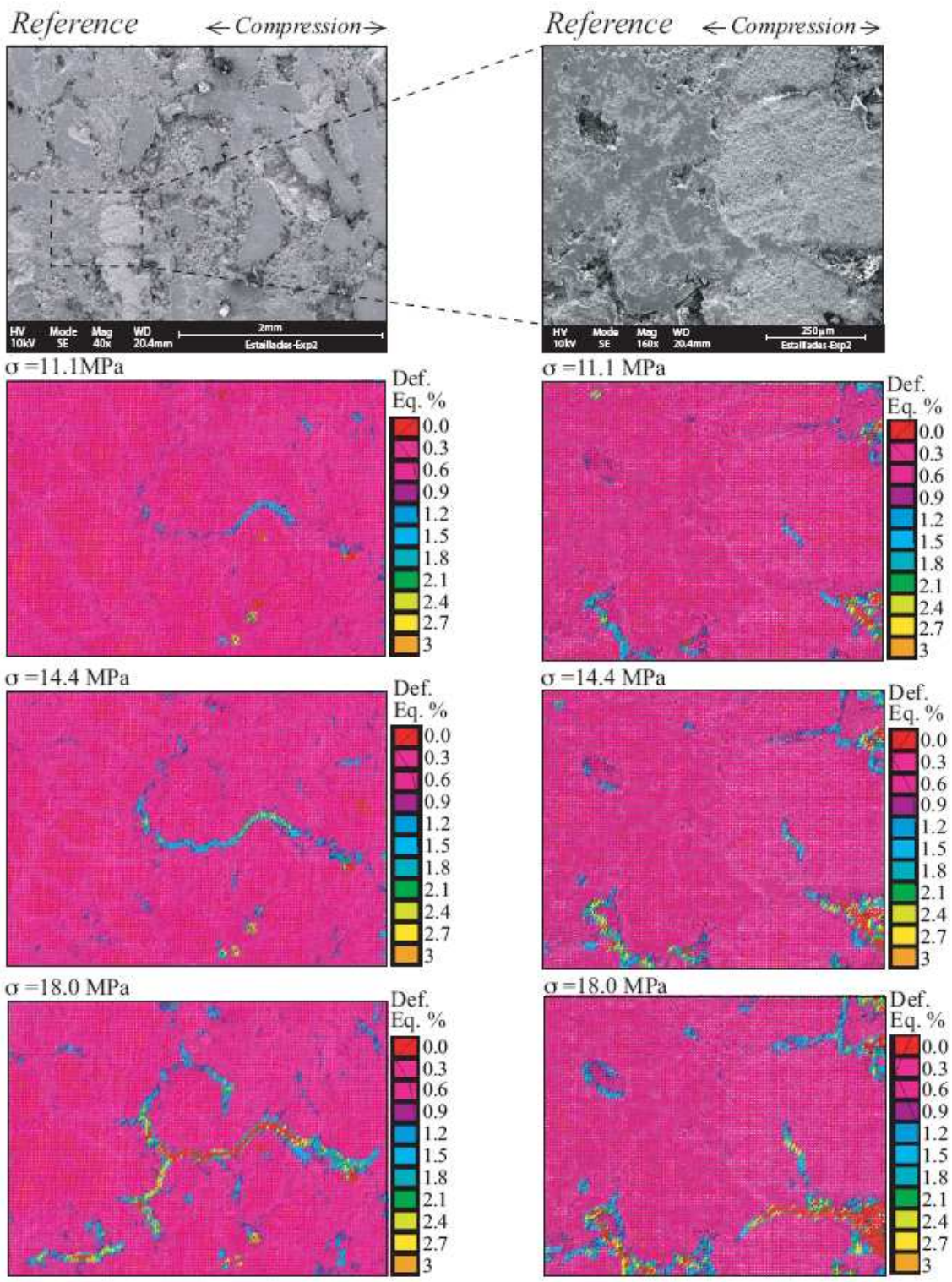

Figure 3. SEM micrographs of the bserved area at two scales and maps of equivalent deformation, corresponding to 3 loading stages. (Measure basis 60 pixels). 


\section{Conclusions}

To our knowledge, this is the first example of DIC applied to the deformation of carbonates. The challenge was the very small deformation at failure which made it crucial to optimize the quality of the picture by improving the local contrast and also by an adequate choice of parameters, such as the size of the domains, so that an optimum can be achieved between precision on strain field computation and accuracy of localization. A quantification of errors and uncertainties has also been done. The different scales of observations have allowed the observation of the various deformation mechanisms and their history in time and space during loading. The two optical scales are consistent and show the importance of heterogeneities at the mesoscopic scale for the early onset of localization as well as the development of a network of microcracks. At the SEM scale, field measurements reveal micromechanisms that cannot be seen by a direct observation of the pictures. It confirms that there is mostly a diffuse accommodation of deformation in the microporous aggregates and an opening of microcracks at the interfaces of dense aggregates and in most porous regions.

\section{References}

1. Chu T., Ranson W., Sutton M. and Peters W. (1985), Applications of DIC techniques to experimental mechanics, Experimental Mechanics, 25,232-244.

2. Bruck H.A., McNeill S.R., Sutton M.A. and Peters W.H. (1989), DIC using NewtonRaphson method of partial differential correction, Experimental Mechanics, 29(3),261-267.

3. Lenoir N., Bornert M., Desrues J., Besuelle P. and Viggiani G. (2007), Volumetric digital image correlation applied to x-ray microtomography images from triaxial compression tests on argillaceous rock, Strain, 43(3),193-205.

4. Valès F. (2008), Modes de déformation et d'endommagement de roches argileuses, $P h D$ thesis, Ecole polytechnique, Palaiseau, France.

5. Valès F., Bornert M., Gharbi H., Nguyen M. D., Eytard J.-C. (2007), Micromechanical investigations of the hydro-mechanical behaviour of argillite rocks, by means of optical full field strain measurement and acoustic emission techniques, Proc. Int. Soc. Rock Mechanics.

6. Noiret A. (2009), 'Contribution a la caractérisation du comportement géomécanique des roches couverture des réservoirs pétroliers', PhD thesis, INPL, Nancy France.

7. Dautriat J. (2009), Comportement hydromécanique de roches réservoirs sous contraintes, PhD thesis, Ecole polytechnique, Palaiseau, France.

8. Yale D. P. and Crawford B. (1998), Plasticity and Permeability in Carbonates: Dependence on Stress Path and Porosity, SPE/ISRM, 47582, 485-494.

9. Doumalin P., Bornert M. and Caldemaison D. (1999), Microextensometry by DIC applied to micromechanical studies using SEM, Proc. Int. Conf. Advanced Technology Experimental Mechanics.

10. Bornert M. (1996), Morphologie microstructurale et comportement mécanique, $P h D$ thesis, Ecole polytechnique, Palaiseau, France.

11. Allais L., Bornert M., Bretheau T. and Caldemaison D. (1994), Experimental characterisation of the local strain field in a heterogeneous elastoplastic material, Acta. Metal. Mater, 42,3865-3880.

12. Doumalin P. (2000), Microextensométrie par corrélation d'images, PhD thesis, Ecole Polytechnique, Palaiseau, France. 